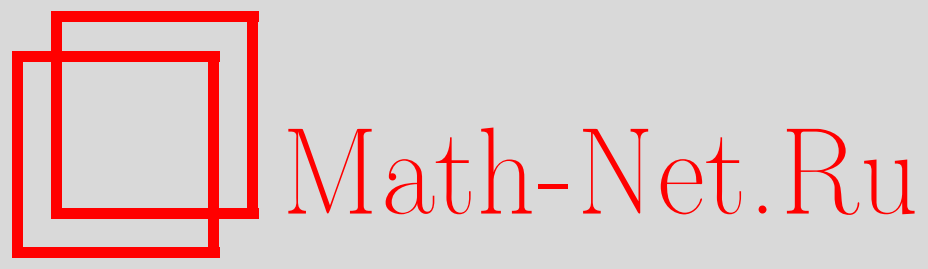

Б. А. Пламеневский, О. В. Сарафанов, О методе вычисления волноводных матриц рассеяния в присутствии точечного спектра, Функи. анализ и его прил., 2014, том 48, выпуск 1, 61-72

DOI: https://doi.org/10.4213/faa3139

Использование Общероссийского математического портала MathNet.Ru подразумевает, что вы прочитали и согласны с пользовательским соглашением http://www . mathnet.ru/rus/agreement

Параметры загрузки:

IP : 18.207 .199 .55

26 апреля 2023 г., 14:43:40

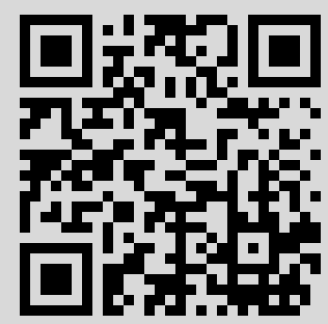




\title{
О методе вычисления волноводных матриц рассеяния в присутствии точечного спектра*
}

\author{
(C) 2014. Б. А. ПЛАМЕНЕВСКИЙ, О. В. САРАФАНОВ
}

\begin{abstract}
Волновод занимает область $G$ в пространстве $\mathbb{R}^{n+1}, n \geqslant 1$, которая имеет несколько цилиндрических выходов на бесконечность. Он описывается общей эллиптической краевой задачей, содержащей спектральный параметр $\mu$ и самосопряженной относительно формулы Грина. В качестве приближения для строки матрицы рассеяния $S(\mu)$ предлагается минимизатор некоторого квадратичного функционала $J^{R}(\cdot, \mu)$. Функционал строится посредством решения вспомогательной краевой задачи в ограниченной области, полученной отрезанием на расстоянии $R$ выходов волновода на бесконечность. Доказывается, что минимизатор $a(R, \mu)$ при $R \rightarrow \infty$ стремится с экспоненциальной скоростью к соответствующей строке матрицы рассеяния равномерно относительно $\mu$ на каждом конечном замкнутом отрезке непрерывного спектра, не содержащем порогов. При этом не исключается присутствие на упомянутом отрезке собственных значений волновода (которым отвечают собственные функции, экспоненциально затухающие на бесконечности).
\end{abstract}

\section{§1. Введение}

Волновод, рассматриваемый в статье, занимает область $G$ в пространстве $\mathbb{R}^{n+1}$ с конечным числом $P$ цилиндрических выходов на бесконечность. Это означает, что вне большого шара с центром в начале координат область $G$ совпадает с объединением непересекающихся полуцилиндров $\Pi_{+}^{1}, \ldots, \Pi_{+}^{P}$; здесь $\Pi_{+}^{p}=\left\{\left(y^{p}, t^{p}\right): y^{p} \in \Omega^{p}, t^{p}>0\right\},\left(y^{p}, t^{p}\right)$ - локальные координаты в $\Pi_{+}^{p}$, и сечение $\Omega^{p}$ цилиндра $\Pi^{p}$ является ограниченной областью в $n$-мерном евклидовом пространстве. Волновод описывается эллиптической краевой задачей $\left\{\mathscr{L}\left(x, D_{x}\right)-\mu, \mathscr{B}\left(x, D_{x}\right)\right\}$ в $G$, где $\mathscr{L}$ - матричный дифференциальный оператор, $\mathscr{B}$ - оператор краевых условий и $\mu-$ спектральный параметр. Оператор $\{\mathscr{L}, \mathscr{B}\}$ самосопряжен относительно формулы Грина. В каждом полуцилиндре $\Pi_{+}^{p}$ коэффициенты операторов $\mathscr{L}$ и $\mathscr{B}$ с экспоненциальной скоростью стабилизируются на бесконечности к функциям, не зависящим от осевой координаты.

Предположим, что отрезок $\left[\mu_{1}, \mu_{2}\right] \subset \mathbb{R}$ принадлежит непрерывному спектру оператора $\{\mathscr{L}, \mathscr{B}\}$. Под этим мы подразумеваем, что для каждого значения $\mu \in\left[\mu_{1}, \mu_{2}\right]$ существуют гладкие решения однородной задачи

$$
\begin{aligned}
\mathscr{L}\left(x, D_{x}\right) u(x)-\mu u(x) & =0, & & x \in G, \\
\mathscr{B}\left(x, D_{x}\right) u(x) & =0, & & x \in \partial G,
\end{aligned}
$$

ограниченные и не принадлежащие (комплексному) пространству $L_{2}(G)$; такие решения называются собственными функциями непрерывного спектра. Не исключаются и собственные значения задачи (1.1) на отрезке $\left[\mu_{1}, \mu_{2}\right]$, которым 
отвечают собственные функции из $L_{2}(G)$. Ввиду упомянутой стабилизации коэффициентов всякая собственная функция из $L_{2}(G)$ исчезает на бесконечности с экспоненциальной скоростью, а собственные значения имеют конечную кратность и на отрезке $\left[\mu_{1}, \mu_{2}\right]$ не сгущаются. Обозначим через $\operatorname{ker}\{\mathscr{L}-\mu, \mathscr{B}\}$ пространство ограниченных решений задачи (1.1). Будем считать, что интервал $\left[\mu_{1}, \mu_{2}\right]$ свободен от пороговых значений спектрального параметра (порогов); определение этого термина дано перед формулой (2.3). Тогда из наших предположений следует, что факторпространство

$$
\operatorname{ker}\{\mathscr{L}-\mu, \mathscr{B}\} /\left(\left(\operatorname{ker}\{\mathscr{L}-\mu, \mathscr{B}\} \cap L_{2}(G)\right)\right.
$$

конечномерное, причем его размерность остается постоянной на отрезке $\left[\mu_{1}, \mu_{2}\right]$; обозначим эту размерность через $M$.

Известно [1], что при каждом $\mu \in\left[\mu_{1}, \mu_{2}\right]$ существуют собственные функции непрерывного спектра $Y_{1}(\cdot, \mu), \ldots, Y_{M}(\cdot, \mu)$ с асимптотикой

$$
Y_{j}(x, \mu)=u_{j}^{+}(x, \mu)+\sum_{k=1}^{M} S_{j k}(\mu) u_{k}^{-}(x, \mu)+O\left(e^{-\varepsilon|x|}\right)
$$

при $|x| \rightarrow \infty$ и $j=1, \ldots, M$; здесь $\varepsilon-$ достаточно малое число, $u_{j}^{+}(\cdot, \mu)$ - «приходящие волны», а $u_{j}^{-}(\cdot, \mu)$ - «уходящие» (точные определения см. в разд. 2.2). Матрица $S(\mu)=\left\|S_{j k}(\mu)\right\|$ унитарная; она называется матрицей рассеяния. Если $\mu$ не является собственным числом задачи (1.1), то функции $Y_{1}(\cdot, \mu), \ldots, Y_{M}(\cdot, \mu)$ определены однозначно и составляют базис в пространстве $\operatorname{ker}\{\mathscr{L}-\mu, \mathscr{B}\}$. Если же число $\mu$ собственное, то $Y_{1}(\cdot, \mu)$ определены с точностью до прибавления собственных функций, матрица рассеяния не зависит от этого произвола, а набору $Y_{1}(\cdot, \mu), \ldots, Y_{M}(\cdot, \mu)$ отвечает базис в упомянутом выше факторпространстве.

Статья посвящена обоснованию метода приближенного вычисления матрицы рассеяния. Подробное описание этого метода дано в разд. 2.2. Коротко говоря, в качестве приближения для $l$-й строки $S_{l}(\mu)=\left(S_{l 1}(\mu), \ldots, S_{l M}(\mu)\right)$ матрицы рассеяния выбирается вектор $a(R, \mu)$, минимизирующий квадратичный функционал $J_{l}^{R}(\cdot, \mu)$. Этот функционал строится посредством решения вспомогательной краевой задачи в ограниченной области $G^{R}$, которая получается из $G$ отрезанием выходов на бесконечность на достаточно далеком расстоянии $R$ от начала координат. В настоящей статье доказывается, что при $R \geqslant R_{0}$ и всех $\mu \in\left[\mu_{1}, \mu_{2}\right]$ справедлива оценка

$$
\left\|a(R, \mu)-S_{l}(\mu)\right\| \leqslant C e^{-\gamma R}
$$

с положительным числом $\gamma$ и постоянной $C$, не зависящими ни от $R$, ни от $\mu$.

Для оператора Гельмгольца в близкой ситуации обсуждаемый метод был предложен в статье [2]. Обоснование метода в [2] опиралось на приведенное без доказательства предложение 3 , которое справедливо лишь при дополнительном условии, отсутствовавшем в предложении 3. Это условие состоит в том, что значение спектрального параметра $\mu$, для которого применяется метод, не должно быть собственным числом основной краевой задачи (в нашем контексте это задача (1.1)); иначе говоря, для $\mu$ не должно существовать собственных функций, экспоненциально затухающих на бесконечности. Для оператора Гельмгольца 
неравенство (1.2) без исключения собственных значений задачи (1.1) из интервала $\left[\mu_{1}, \mu_{2}\right]$ было доказано в [3]. В настоящей статье неравенство (1.2) распространяется на общие самосопряженные эллиптические задачи. Предлагаемое обоснование метода является новым и для оператора Гельмгольца; оно проще рассуждений, использованных в [3]. В отличие от [3], здесь мы не строим приближенное решение вспомогательной задачи в области $G^{R}$, а применяем простую оценку ее решений, равномерную относительно $R \geqslant R_{0}$ и $\mu \in\left[\mu_{1}, \mu_{2}\right]$ и безразличную к присутствию на отрезке $\left[\mu_{1}, \mu_{2}\right]$ собственных значений задачи (1.1). Впрочем, подход, изложенный в статье [3], объясняет (и, быть может, более выразительно) механизм метода вычисления матрицы рассеяния с другой точки зрения.

В неоднородных волноводах сложной геометрии, как правило, имеются ловушечные моды, т. е. экспоненциально убывающие собственные функции. Поэтому возможность не заботиться в приложениях о ловушечных модах оказывается важным достоинством метода.

Точное определение матрицы рассеяния, подробное описание метода ее вычисления и формулировка основной теоремы 2.1 даны в §2. Разрешимость вспомогательной задачи в области $G^{R}$ обсуждается в $\S 3$. Наконец, оправдание метода завершается в $\S 4$ доказательством теоремы 2.1.

\section{§2. Метод вычисления матрицы рассеяния}

2.1. Матрица рассеяния. В области $G$ введем формально самосопряженную $(k \times k)$-матрицу $\left\|\mathscr{L}_{i j}\left(x, D_{x}\right)\right\|$ дифференциальных операторов, где ord $\mathscr{L}_{i j}=$ $\tau_{i}+\tau_{j}, \tau_{j}$ - неотрицательные целые числа и $\tau_{1}+\cdots+\tau_{k}=m$. Будем считать, что справедлива формула Грина

$$
(\mathscr{L} u, v)_{G}+(\mathscr{B} u, \mathscr{Q} v)_{\partial G}=(u, \mathscr{L} v)_{G}+(\mathscr{Q} u, \mathscr{B} v)_{\partial G}
$$

для всех $u, v \in C_{c}^{\infty}\left(\bar{G}, \mathbb{C}^{k}\right),(m \times k)$-матрицы $\mathscr{B}=\left\|\mathscr{B}_{q j}\right\|$ и $\mathscr{Q}=\left\|\mathscr{Q}_{q j}\right\|$ состоят из дифференциальных операторов, причем ord $\mathscr{B}_{q j}=\sigma_{q}+\tau_{j}$, где $\sigma_{q}$ - целое число, ord $\mathscr{B}_{q j}+\operatorname{ord} \mathscr{Q}_{q i} \leqslant \tau_{j}+\tau_{i}-1$ и по определению $\mathscr{B}_{q j}=0$ в случае, когда $\sigma_{q}+\tau_{j}<0$; через $(\cdot, \cdot)_{G}$ и $(\cdot, \cdot)_{\partial G}$ обозначены скалярные произведения на комплексных пространствах $L_{2}(G)$ и $L_{2}(\partial G)$. Коэффициенты операторов $\mathscr{L}$, $\mathscr{B}$ и $\mathscr{Q}$ предполагаются комплексными и гладкими. Мы считаем, что оператор $\{\mathscr{L}, \mathscr{B}\}$ краевой задачи в области $G$ эллиптический по Дуглису-Ниренбергу.

Опишем поведение коэффициентов операторов $\mathscr{L}$ и $\mathscr{B}$ на бесконечности. Пусть $\left\{L^{p}, B^{p}\right\}$ - оператор эллиптической задачи в цилиндре $\Pi^{p}=\Omega^{p} \times \mathbb{R}$ с коэффициентами, не зависящими от $t^{p} \in \mathbb{R}$ и гладкими в $\overline{\Omega^{p}}$, причем ord $L_{i j}^{p}=$ $\operatorname{ord} \mathscr{L}_{i j}$ и ord $B_{i j}^{p}=\operatorname{ord} \mathscr{B}_{i j}$. Будем считать, что всякий коэффициент а оператора $\{\mathscr{L}, \mathscr{B}\}$ удовлетворяет в $\Pi_{+}^{p}$ соотношению

$$
D^{\alpha}\left(a\left(y^{p}, t^{p}\right)-a^{p}\left(y^{p}\right)\right)=O\left(\exp \left(-\delta t^{p}\right)\right)
$$

при $t^{p} \rightarrow \infty$ и всех мультииндексах $\alpha$, где $a^{p}-$ соответствующий коэффициент оператора $\left\{L^{p}, B^{p}\right\}, \delta$ - некоторое положительное число, $i^{|\alpha|} D^{\alpha}-$ производная по переменным $\left(y^{p}, t^{p}\right)$. Из (2.1) вытекает, что в каждом цилиндре $\Pi^{p}$ справедлива формула Грина, которая получается из (2.1) заменой $G$ на $\Pi^{p}$ и операторов $\mathscr{L}, \mathscr{B}, \mathscr{Q}$ на $L^{p}, B^{p}, Q^{p}$. 
В области $\Omega=\Omega^{p}$ введем операторный пучок

$$
\mathbb{C} \ni \lambda \mapsto \mathfrak{A}^{p}(\lambda, \mu)=\left\{L^{p}(\lambda)-\mu I, B^{p}(\lambda)\right\},
$$

где $L^{p}(\lambda)=L^{p}\left(y, D_{y}, \lambda\right), B^{p}(\lambda)=B^{p}\left(y, D_{y}, \lambda\right)$. Таким образом, мы имеем пучки $\mathfrak{A}^{1}(\cdot, \mu), \ldots, \mathfrak{A}^{P}(\cdot, \mu)$. Спектр пучка составляют изолированные собственные значения. Каждая полоса $\{\lambda \in \mathbb{C}:|\operatorname{Im} \lambda|<h<\infty\}$ содержит разве лишь конечное число собственных значений (см. [4]).

Уточним предположения относительно отрезка $\left[\mu_{1}, \mu_{2}\right]$. Будем считать, что при $\mu \in\left[\mu_{1}, \mu_{2}\right]$ каждому вещественному собственному числу пучка $\lambda \mapsto \mathfrak{A}^{p}(\lambda, \mu)$, $p=1, \ldots, P$, отвечают лишь собственные векторы, присоединенных векторов нет, а сумма кратностей вещественных собственных чисел пучка $\mathfrak{A}^{p}(\cdot, \mu)$ остается постоянной при $\mu \in\left[\mu_{1}, \mu_{2}\right]$; это и означает, что порогов на отрезке $\left[\mu_{1}, \mu_{2}\right]$ нет. Известно, что сумма кратностей вещественных собственных чисел пучка четная (см. [1]).

Перенумеруем все вещественные собственные числа пучка $\mathfrak{A}^{p}(\cdot, \mu)$ с учетом кратности. Пусть $\lambda_{1}, \ldots, \lambda_{2 M^{p}}$ - все такие числа и $\varphi_{1}, \ldots, \varphi_{2 M^{p}}$ - отвечающие этим числам собственные векторы. Функции

$$
u_{k}(y, t)=\exp \left(i \lambda_{k} t\right) \varphi_{k}(y)
$$

удовлетворяют однородной задаче

$$
\begin{array}{ll}
\left(L^{p}\left(y, D_{y}, D_{t}\right)-\mu\right) v(y, t)=0, & (y, t) \in \Pi^{p}, \\
B^{p}\left(y, D_{y}, D_{t}\right) v(y, t)=0, & (y, t) \in \partial \Pi^{p} .
\end{array}
$$

Введем форму

$$
q^{p}(u, v)=\left(L^{p} u, v\right)_{\Pi^{p}}+\left(B^{p} u, Q^{p} v\right)_{\partial \Pi^{p}}-\left(u, L^{p} v\right)_{\Pi^{p}}-\left(Q^{p} u, B^{p} v\right)_{\partial \Pi^{p}} .
$$

Если $u, v \in C_{c}^{\infty}\left(\overline{\Pi^{p}}\right)$, то $q^{p}(u, v)=0$. Пусть $\chi \in C^{\infty}(\mathbb{R}), \chi(t)=1$ при $t \geqslant 2$ и $\chi(t)=0$ при $t \leqslant 1$. Форма $q^{p}$ распространяется на пары $\left\{\chi u_{j}, \chi u_{k}\right\}$, причем собственные векторы $\left\{\varphi_{k}\right\}$ могут быть выбраны так, что выполняются условия $q^{p}\left(\chi u_{j}, \chi u_{k}\right)= \pm i \delta_{j k}$, где знак не может быть выбран произвольно, а определяется собственным числом $\lambda_{k}$.

Продолжим нулем функции $\chi u_{k}$ из $\Pi_{+}^{p}$ на область $G$. Введем линейное пространство $E$ таких $w \in C^{\infty}(\bar{G})$, что $D^{\alpha} w(x)=O(\exp (-\beta|x|))$ при $|x| \rightarrow+\infty$ для всех мультииндексов $\alpha$ и некоторого $\beta>0$ (нам удобно принять, что $\beta$ совпадает с числом $\gamma$ из (2.8)). Через $\mathscr{W}$ обозначим линейную оболочку функций вида $\chi u_{k}+w$, где $w \in E$. Тогда форма

$$
q(u, v)=(\mathscr{L} u, v)_{G}+(\mathscr{B} u, \mathscr{Q} v)_{\partial G}-(u, \mathscr{L} v)_{G}-(\mathscr{Q} u, \mathscr{B} v)_{\partial G}
$$

принимает конечные значения для любых $u, v \in \mathscr{W}$. Очевидно, что $q(u, v)=$ $-\overline{q(v, u)}$ и $q(u, u) \in i \mathbb{R}$. Выберем элемент $u \in \mathscr{W}$, представимый в виде $u=$ $U^{r}+w$, где $U^{r}$ - линейная комбинация функций вида $\chi u_{k}$ с носителями в $\Pi_{+}^{r}$, а $w \in E$. По определению $u$ является приходящей (уходящей) волной, если $i q(u, u)>0(i q(u, u)<0)$. Как показано в [1], в факторпространстве $\mathscr{W} / E$ можно выбрать базис

$$
u_{1}^{+}, \ldots, u_{M}^{+}, u_{1}^{-}, \ldots, u_{M}^{-}, \quad M=\sum_{p=1}^{P} M^{p},
$$


подчиненный условиям

$$
q\left(u_{j}^{ \pm}, u_{k}^{ \pm}\right)=\mp i \delta_{j k}, \quad q\left(u_{j}^{ \pm}, u_{k}^{\mp}\right)=0, \quad j, k=1, \ldots, M ;
$$

здесь $u_{1}^{+}, \ldots, u_{M}^{+}-$приходящие волны и $u_{1}^{-}, \ldots, u_{M}^{-}-$уходящие волны.

Обратимся к задаче

$$
\mathscr{L}\left(x, D_{x}\right) u(x)-\mu u(x)=0, \quad x \in G ; \quad \mathscr{B}\left(x, D_{x}\right) u(x)=0, \quad x \in \partial G .
$$

Пусть $0<\gamma<\delta$, где $\delta$ - число из формулы $(2.2)$, и пусть полоса $\{\lambda \in \mathbb{C}:$ $|\operatorname{Im} \lambda| \leqslant \gamma\}$ содержит лишь вещественные собственные числа пучков $\mathfrak{A}^{p}(\cdot, \mu)$, где $p=1, \ldots, P$ и $\mu \in\left[\mu_{1}, \mu_{2}\right]$. Известно [1], что существуют решения $Y_{1}(\cdot, \mu), \ldots$, $Y_{M}(\cdot, \mu)$ задачи $(2.7)$, гладкие в $\bar{G}$ и допускающие при больших $|x|$ представления

$$
Y_{j}(x, \mu)=u_{j}^{+}(x)+\sum_{k=1}^{M} S_{j k}(\mu) u_{k}^{-}(x)+O\left(e^{-\gamma|x|}\right) .
$$

Если число $\mu$ не является собственным для задачи $(2.7)$, то функции $Y_{j}(\cdot, \mu)$ определены однозначно и образуют базис в пространстве ограниченных решений однородной задачи (2.7) (собственных функций непрерывного спектра). В противном случае всякое решение $Y_{j}(\cdot, \mu)$ определено с точностью до слагаемого - собственной функции из $L_{2}(G)$ задачи $(2.7)$. Тогда любое ограниченное решение задачи (2.7) представляется линейной комбинацией функций $Y_{j}(\cdot, \mu)$ с точностью до собственной функции из $L_{2}(G)$.

Матрица $S(\mu)=\left\|S_{j k}(\mu)\right\|_{j, k=1}^{M}$ в (2.8) определена однозначно при всех $\mu \in$ $\left[\mu_{1}, \mu_{2}\right]$; она не зависит от возможного произвола в определении $Y_{j}(\cdot, \mu)$ для $\mu$, оказывающихся собственными значениями. Матрица $S(\mu)$ называется матрицей рассеяния; она унитарная при всех $\mu$.

2.2. Метод вычисления матрицы рассеяния. Введем обозначения

$$
\Pi_{+}^{p, R}=\left\{\left(y^{p}, t^{p}\right) \in \Pi^{p}: t^{p}>R\right\}, \quad G^{R}=G \backslash \bigcup_{p=1}^{P} \Pi_{+}^{p, R}
$$

для больших $R$. Тогда $\partial G^{R} \backslash \partial G=\Gamma^{R}=\bigcup_{p} \Gamma^{p, R}$, где $\Gamma^{p, R}=\left\{\left(y^{p}, t^{p}\right) \in \Pi^{p}:\right.$ $\left.t^{p}=R\right\}$. При больших $R$ имеет место равенство

$$
\begin{aligned}
(\mathscr{L} u, v)_{G^{R}}+(\mathscr{B} u, \mathscr{Q} v)_{\partial G^{R} \backslash \Gamma^{R}}+(\mathscr{N} u, \mathscr{D} v)_{\Gamma^{R}} & \\
& =(u, \mathscr{L} v)_{G^{R}}+(\mathscr{Q} u, \mathscr{B} v)_{\partial G^{R} \backslash \Gamma^{R}}+(\mathscr{D} u, \mathscr{N} v)_{\Gamma^{R}}
\end{aligned}
$$

где $\mathscr{D}$ и $\mathscr{N}$ - матрицы дифференциальных операторов размера $m \times k, \mathscr{D}-$ система Дирихле (см. [5]). Примером системы Дирихле является матрица из $m$ строк вида $e^{(j)} \partial_{\nu}^{h}$, где $j=1, \ldots, k, h=1, \ldots, \tau_{j}-1, e^{(j)}=\left(\delta_{1, j}, \ldots, \delta_{k, j}\right)$, а $\nu-$ внешняя нормаль к $\Gamma^{R}$. Оператор $\mathscr{N}$ при выбранном операторе $\mathscr{D}$ определяется требованием, чтобы выполнялась формула Грина (2.9).

Будем искать строку $\left(S_{l 1}, \ldots, S_{l M}\right)$ матрицы рассеяния $S=S(\mu)$. В качестве приближения для этой строки возьмем вектор, минимизирующий некоторый 
квадратичный функционал. Для построения функционала рассмотрим задачу

$$
\begin{array}{ll}
\left(\mathscr{L}\left(x, D_{x}\right)-\mu\right) \mathscr{X}_{l}^{R}=0, & x \in G^{R}, \\
\mathscr{B}\left(x, D_{x}\right) \mathscr{X}_{l}^{R}=0, & x \in \partial G^{R} \backslash \Gamma^{R}, \\
\left(\mathscr{N}\left(x, D_{x}\right)+i \zeta \mathscr{D}\left(x, D_{x}\right)\right) \mathscr{X}_{l}^{R} & \\
\quad=\left(\mathscr{N}\left(x, D_{x}\right)+i \zeta \mathscr{D}\left(x, D_{x}\right)\right)\left(u_{l}^{+}+\sum_{j=1}^{M} a_{j} u_{j}^{-}\right), & x \in \Gamma^{R},
\end{array}
$$

где $\zeta \in \mathbb{R} \backslash\{0\}$, а числа $a_{1}, \ldots, a_{M}$ комплексные.

Объясним происхождение этой задачи. Решение $Y_{l}$ однородной задачи (2.7) удовлетворяет первым двум уравнениям (2.10). Асимптотику (2.8) можно дифференцировать, поэтому

$$
(\mathscr{N}+i \zeta \mathscr{D}) Y_{l}=(\mathscr{N}+i \zeta \mathscr{D})\left(u_{l}^{+}+\sum_{j=1}^{M} a_{j} u_{j}^{-}\right)+O\left(e^{-\gamma R}\right)
$$

при $a_{j}=S_{l j}$. Таким образом, функция $Y_{l}$ оставляет в последнем уравнении (2.10) экспоненциально малую невязку. В качестве приближения для строки $\left(S_{l 1}, \ldots, S_{l M}\right)$ возьмем вектор $a^{0}(R)=\left(a_{1}^{0}(R), \ldots, a_{M}^{0}(R)\right)$, доставляющий минимум функционала

$$
J_{l}^{R}\left(a_{1}, \ldots, a_{M}\right)=\left\|\mathscr{D}\left(\mathscr{X}_{l}^{R}-u_{l}^{+}-\sum_{j=1}^{M} a_{j} u_{j}^{-}\right) ; L_{2}\left(\Gamma^{R}\right)\right\|^{2},
$$

где $\mathscr{X}_{l}^{R}$ - решение задачи (2.10). Можно ожидать, что $a_{j}^{0}(R) \rightarrow S_{l j}$ с экспоненциальной скоростью при $R \rightarrow \infty$ и $j=1, \ldots, M$. Чтобы выписать зависимость $\mathscr{X}_{l}^{R}$ от параметров $a_{1}, \ldots, a_{M}$, рассмотрим задачи

$$
\begin{array}{ll}
\left(\mathscr{L}\left(x, D_{x}\right)-\mu\right) v_{j}^{ \pm}=0, & x \in G^{R}, \\
\mathscr{B}\left(x, D_{x}\right) v_{j}^{ \pm}=0, & x \in \partial G^{R} \backslash \Gamma^{R}, \\
\left(\mathscr{N}\left(x, D_{x}\right)+i \zeta \mathscr{D}\left(x, D_{x}\right)\right) v_{j}^{ \pm} & \\
\quad=\left(\mathscr{N}\left(x, D_{x}\right)+i \zeta \mathscr{D}\left(x, D_{x}\right)\right) u_{j}^{ \pm}, & x \in \Gamma^{R}, j=1, \ldots, M .
\end{array}
$$

Выразим $\mathscr{X}_{l}^{R}$ через решения $v_{j}^{ \pm}=v_{j, R}^{ \pm}$задач $(2.12)$. Имеем $\mathscr{X}_{l}^{R}=v_{l, R}^{+}+$ $\sum_{j} a_{j} v_{j, R}^{-}$. Введем $(M \times M)$-матрицы с элементами

$$
\begin{aligned}
\mathscr{E}_{i j}^{R} & =\left(\mathscr{D}\left(v_{i}^{-}-u_{i}^{-}\right), \mathscr{D}\left(v_{j}^{-}-u_{j}^{-}\right)\right)_{\Gamma^{R}}, \\
\mathscr{F}_{i j}^{R} & =\left(\mathscr{D}\left(v_{i}^{+}-u_{i}^{+}\right), \mathscr{D}\left(v_{j}^{-}-u_{j}^{-}\right)\right)_{\Gamma^{R}} .
\end{aligned}
$$

Положим, кроме того,

$$
\mathscr{G}_{i}^{R}=\left(\mathscr{D}\left(v_{i}^{+}-u_{i}^{+}\right), \mathscr{D}\left(v_{i}^{+}-u_{i}^{+}\right)\right)_{\Gamma^{R}} .
$$

Теперь функционал (2.11) можно записать в виде

$$
J_{l}^{R}(a)=\left\langle a \mathscr{E}^{R}, a\right\rangle+2 \operatorname{Re}\left\langle\mathscr{F}_{l}^{R}, a\right\rangle+\mathscr{G}_{l}^{R},
$$

где $\mathscr{F}_{l}^{R}$ - строка с номером $l$ матрицы $\mathscr{F}^{R}$ и $\langle\cdot, \cdot\rangle-$ скалярное произведение в $\mathbb{C}^{M}$. Минимум реализуется на векторе $a^{0}$ (строке), удовлетворяющем системе $a^{0}(R) \mathscr{E}^{R}+\mathscr{F}_{l}^{R}=0$. Итак, в качестве приближения $S^{R}(\mu)$ для матрицы рассеяния $S(\mu)$ выступает решение уравнения $S^{R} \mathscr{E}^{R}+\mathscr{F}^{R}=0$. 
Остальная часть статьи посвящена доказательству следующей теоремы, которая является основным результатом работы.

Теорема 2.1. Пусть $\zeta$ - любое фиксированное ненулевое вещественное число. Пусть отрезок $\left[\mu_{1}, \mu_{2}\right]$ непрерьвного спектра задачи (2.7) удовлетворяет условиям, указанным перед формулой (2.3) (иными словами, интервал $\left[\mu_{1}, \mu_{2}\right]$ свободен от пороговых значений спектрального параметра $\mu)$. Тогда для всех $\mu \in\left[\mu_{1}, \mu_{2}\right]$ u $R>R_{0}$, где $R_{0}$ - достаточно большое число, существует единственный минимизатор $a^{0}(R, \mu)=\left(a_{1}^{0}(R, \mu), \ldots, a_{M}^{0}(R, \mu)\right)$ функщионала $J_{l}^{R}(\cdot ; \mu)$ из (2.11). Справедливы неравенства

$$
\left|a_{j}^{0}(R, \mu)-S_{l j}(\mu)\right| \leqslant C e^{-\gamma R}, \quad j=1, \ldots, M,
$$

где $\gamma$ - то же число, что и в (2.8), а постоянная $C$ не зависит ни от $\mu$, ни om $R$.

\section{§3. Задача в области $G^{R}$}

Введем краевую задачу

$$
\begin{aligned}
\mathscr{L}\left(x, D_{x}\right) u(x)-\mu u(x) & =f(x), & & x \in G^{R}, \\
\mathscr{B}\left(x, D_{x}\right) u(x) & =g(x), & & x \in \partial G^{R} \backslash \Gamma^{R}, \\
\left(\mathscr{N}\left(x, D_{x}\right)+i \zeta \mathscr{D}\left(x, D_{x}\right)\right) u(x) & =h(x), & & x \in \Gamma^{R},
\end{aligned}
$$

где $\zeta \in \mathbb{R} \backslash\{0\}$ и $\mu \in \mathbb{R}$. Граница $\partial G^{R}$ содержит ребро $\partial \Gamma^{R}$ при $\operatorname{dim} G>2$ или угловые точки при $\operatorname{dim} G=2 ;$ на $\partial \Gamma^{R}$ меняются граничные условия. Для исследования задачи (3.1) можно использовать обычную схему теории эллиптических краевых задач в областях с кусочно гладкой границей (см., например, [6], [7], [1], [8]). В отличие от гладкой ситуации выбор функциональных пространств для краевой задачи не является универсальным и требует учета специфических свойств решений вблизи ребер (иногда привлекаются пространства с весовыми нормами, где вес есть степень расстояния до ребра). Для ряда классических задач математической физики нужные пространства известны. По этим причинам здесь мы ограничимся некоторыми соображениями, связанными со спецификой задачи (3.1), примером и постулированием свойств функциональных пространств без их детального определения.

Для того чтобы обеспечить замкнутость образа оператора задачи (3.1), функциональные пространства выбираются так, что оператор задачи оказывается фредгольмовым (т. е. его образ замкнут, а ядро и коядро конечномерны). Тогда тривиальность ядра и коядра необходима и достаточна для существования и единственности решения задачи при любой правой части. Для доказательства этой тривиальности понадобится следующая лемма.

Лемма 3.1. Пусть $u$ - решение задачи (3.1) при $f=0$ u $g=0$. Тогда

$$
\left\|\mathscr{D} u ; L_{2}\left(\Gamma^{R}\right)\right\| \leqslant \frac{1}{|\zeta|}\left\|h ; L_{2}\left(\Gamma^{R}\right)\right\| .
$$

Доказательство. Из равенства (2.9) следует формула Грина

$$
\begin{aligned}
& (\mathscr{L} u, v)_{G^{R}}+(\mathscr{B} u, \mathscr{Q} v)_{\partial G^{R} \backslash \Gamma^{R}}-(u, \mathscr{L} v)_{G^{R}}-(\mathscr{Q} u, \mathscr{B} v)_{\partial G^{R} \backslash \Gamma^{R}} \\
& =-((\mathscr{N}+i \zeta \mathscr{D}) u, \mathscr{D} v)_{\Gamma^{R}}+(\mathscr{D} u,(\mathscr{N}+i \zeta \mathscr{D}) v)_{\Gamma^{R}}-2 i \zeta(\mathscr{D} u, \mathscr{D} v)_{\Gamma^{R}} .
\end{aligned}
$$


Положим в ней $v=u$ :

$$
0=-(h, \mathscr{D} u)_{\Gamma^{R}}+(\mathscr{D} u, h)_{\Gamma^{R}}-2 i \zeta\left\|\mathscr{D} u ; L_{2}\left(\Gamma^{R}\right)\right\|^{2} .
$$

Отсюда следует, что

$$
2|\zeta|\left\|\mathscr{D} u ; L_{2}\left(\Gamma^{R}\right)\right\|^{2}=\left|(h, \mathscr{D} u)_{\Gamma^{R}}-(\mathscr{D} u, h)_{\Gamma^{R}}\right| \leqslant 2\left\|\mathscr{D} u ; L_{2}\left(\Gamma^{R}\right)\right\|\left\|h ; L_{2}\left(\Gamma^{R}\right)\right\|,
$$

и мы приходим к неравенству (3.2).

Вернемся к проверке тривиальности ядра. Пусть $u$ - решение однородной задачи (3.1). Из (3.2) и однородного краевого условия $\left(\mathscr{N}\left(x, D_{x}\right)+i \zeta \mathscr{D}\left(x, D_{x}\right)\right) u(x)$ $=0$ при $x \in \Gamma^{R}$ вытекает, что решение $u$ имеет нулевые данные Коши на $\Gamma^{R}$. Если коэффициенты оператора достаточно гладкие для применимости теоремы о единственности продолжения решения (см. [9, часть II, §1.5]), то мы получаем тривиальность ядра. Аналогичные соображения для сопряженной задачи доставляют тривиальность коядра (предполагается, конечно, что функциональные пространства, в которых рассматривается задача, допускают такие соображения). Мы реализуем этот план в простой ситуации следующего примера.

Пример. Будем считать, что $\operatorname{dim} G=2$ и рассмотрим задачу

$$
\begin{aligned}
(\Delta-\mu) u(x) & =f(x), & & x \in G^{R}, \\
u(x) & =g(x), & & x \in \partial G^{R} \backslash \Gamma^{R}, \\
\partial_{\nu} u(x)+i \zeta u(x) & =h(x), & & x \in \Gamma^{R} .
\end{aligned}
$$

С угловыми точками границы $\partial G^{R}$ свяжем задачу с комплексным параметром (операторный пучок)

$$
\begin{aligned}
\left(\partial_{\omega}^{2}-\lambda^{2}\right) v(\omega) & =p(\omega), \quad \omega \in(0, \pi / 2), \\
v(0)=v^{\prime}(\pi / 2) & =0 .
\end{aligned}
$$

Спектр этой задачи состоит из простых собственных чисел $\lambda_{q}=(2 q+1) i$, где $q=0, \pm 1, \ldots$ Числу $\lambda_{q}$ отвечает собственная функция $\varphi_{q}(\omega)=\sin (2 q+1) \omega$.

Введем пространство $V_{\beta}^{l}\left(G^{R}\right)$ с нормой

$$
\left\|u ; V_{\beta}^{l}\left(G^{R}\right)\right\|=\left(\sum_{|\alpha| \leq l} \int_{G^{R}} r^{2(\beta-l+|\alpha|)}\left|D_{x}^{\alpha} u(x)\right|^{2} d x\right)^{1 / 2},
$$

где $\beta \in \mathbb{R}, l=0,1, \ldots$ и через $r$ обозначена функция, совпадающая вблизи угловой точки с расстоянием до этой точки, вне окрестности угловых точек равная единице, гладкая и строго положительная на $\overline{G^{R}}$ (кроме угловых точек). Пусть $V_{\beta}^{l-1 / 2}\left(\partial G^{R} \backslash \Gamma^{R}\right)$ и $V_{\beta}^{l-1 / 2}\left(\Gamma^{R}\right)$ при $l=1,2, \ldots$ обозначают пространства следов функций из $V_{\beta}^{l}\left(G^{R}\right)$ на $\partial G^{R} \backslash \Gamma^{R}$ и $\Gamma^{R}$ соответственно.

Оператор $\mathscr{A}^{R}(\mu)$ задачи (3.3) осуществляет непрерывное отображение

$$
V_{\beta}^{2}\left(G^{R}\right) \ni u \mapsto \mathscr{A}^{R}(\mu) u=\{f, g, h\} \in V_{\beta}^{0}\left(G^{R}\right) \times V_{\beta}^{3 / 2}\left(\partial G^{R} \backslash \Gamma^{R}\right) \times V_{\beta}^{1 / 2}\left(\Gamma^{R}\right) .
$$

Известно, что оператор (3.5) фредгольмов в том и только в том случае, когда число $\beta-1$ не совпадает ни с одним из чисел $\operatorname{Im} \lambda_{q}$, т. е. оператор фредгольмов, когда $\beta$ не является четным. Если функция $w$ удовлетворяет однородной задаче (3.3) и $w \in V_{\beta}^{2}\left(G^{R}\right)$ при некотором $\beta \in(2 q, 2 q+2)$ и каком-нибудь целом $q$, 
то вблизи угловой точки $w(x)=r^{2 q+1} \sin (2 q+1) \omega+O\left(r^{2 q+2-\varepsilon}\right)$, где $r, \omega-$ полярные координаты с центром в угловой точке, $C-$ постоянная, а $\varepsilon-$ любое положительное число, подчиненное неравенству $\varepsilon<1$. Поэтому для любого элемента из ядра оператора (3.5) при $\beta \in(0,2)$ верна оценка $(3.2)$, из которой следует, что $\left\|w ; L_{2}\left(\Gamma^{R}\right)\right\|=0$. Значит, ядро тривиально при $\beta \in(0,2)$, а потому и при всех $\beta<2$.

Теперь займемся коядром. Обозначим через $V_{-\beta}^{-l}\left(G^{R}\right)$ пространство, сопряженное с $V_{\beta}^{l}\left(G^{R}\right)$ относительно скалярного произведения в $L_{2}\left(G^{R}\right)$, а через $V_{-\beta}^{-1 / 2}\left(\Gamma^{R}\right)$ - пространство, сопряженное с $V_{\beta}^{1 / 2}\left(\Gamma^{R}\right)$ относительно двойственности в $L_{2}\left(\Gamma^{R}\right)$; аналогичный смысл имеет $V_{-\beta}^{-3 / 2}\left(\partial G^{R} \backslash \Gamma^{R}\right)$. Далее, пусть $\mathscr{A}^{R}(\mu)^{*}$ - оператор, сопряженный с оператором (3.5),

$$
\mathscr{A}^{R}(\mu)^{*}: V_{-\beta}^{0}\left(G^{R}\right) \times V_{-\beta}^{-3 / 2}\left(\partial G^{R} \backslash \Gamma^{R}\right) \times V_{-\beta}^{-1 / 2}\left(\Gamma^{R}\right) \rightarrow V_{-\beta}^{-2}\left(G^{R}\right) .
$$

Коядро оператора (3.5) совпадает с ядром оператора (3.6). Согласно известным результатам о регулярности решений эллиптических задач, для всякого элемента $\{u, v, w\}$ ядра оператора (3.6) справедливо включение

$$
\{u, v, w\} \in V_{2-\beta}^{2}\left(G^{R}\right) \times V_{2-\beta}^{1 / 2}\left(\partial G^{R} \backslash \Gamma^{R}\right) \times V_{2-\beta}^{3 / 2}\left(\Gamma^{R}\right),
$$

причем $u$ удовлетворяет однородной краевой задаче $(3.3)$ с заменой $\partial_{\nu}+i \zeta$ на $\partial_{\nu}-i \zeta$ в краевом условии на $\Gamma^{R}$, а $v$ и $w$ определяются равенствами

$$
v(x)=-\partial_{\nu} u(x), \quad x \in \partial G^{R} \backslash \Gamma^{R} ; \quad w(x)=u(x), \quad x \in \Gamma^{R} .
$$

Приведенное выше обсуждение тривиальности ядра оператора (3.5) не зависит от знака $\zeta$. Поэтому, учитывая (3.7), получаем, что $u=0$ при всех $\beta$, таких, что $2-\beta<2$. В силу (3.8) при таких же $\beta$ имеем $v=0$ и $w=0$. Итак, если $\beta \in(0,2)$, то тривиальны и ядро, и коядро оператора (3.5). Значит, при $\beta \in(0,2)$ и всех $\mu$ и $\zeta \neq 0$ оператор (3.5) - изоморфизм. Можно показать, что при четном $\beta$ образ этого оператора не замкнут, при $\beta<0$ оператор имеет нетривиальное коядро, а при $\beta>2$ - нетривиальное ядро.

\section{§4. Обоснование метода вычисления матрицы рассеяния}

Для обоснования метода осталось проверить невырожденность матрицы $\mathscr{E} R$ с элементами (2.13) и стабилизацию минимизатора $a^{0}(R)$ функционала $(2.11)$ при $R \rightarrow \infty$ к $l$-й строке матрицы рассеяния. Все оценки этого параграфа выполняются равномерно относительно $\mu \in\left[\mu_{1}, \mu_{2}\right]$.

Лемма 4.1. Пусть $u_{j}^{ \pm}$- приходящие и уходящие волны $(2.5)$, подчиненнье соотношениям (2.6). Тогда

$$
\begin{gathered}
\left(\mathscr{N} u_{j}^{ \pm}, \mathscr{D} u_{k}^{ \pm}\right)_{\Gamma^{R}}-\left(\mathscr{D} u_{j}^{ \pm}, \mathscr{N} u_{k}^{ \pm}\right)_{\Gamma^{R}}=\mp i \delta_{j k}+O\left(e^{-\gamma R}\right), \\
\left(\mathscr{N} u_{j}^{ \pm}, \mathscr{D} u_{k}^{\mp}\right)_{\Gamma^{R}}-\left(\mathscr{D} u_{j}^{ \pm}, \mathscr{N} u_{k}^{\mp}\right)_{\Gamma^{R}}=O\left(e^{-\gamma R}\right)
\end{gathered}
$$

при $R \rightarrow+\infty$; здесъ $\gamma-$ то же положительное число, что и в бормуле (2.8).

Доказательство. Согласно (2.9),

$$
\begin{aligned}
(\mathscr{N} u, \mathscr{D} v)_{\Gamma^{R}} & -(\mathscr{D} u, \mathscr{N} v)_{\Gamma^{R}} \\
& =(u, \mathscr{L} v)_{G^{R}}+(\mathscr{Q} u, \mathscr{B} v)_{\partial G^{R} \backslash \Gamma^{R}}-(\mathscr{L} u, v)_{G^{R}}-(\mathscr{B} u, \mathscr{Q} v)_{\partial G^{R} \backslash \Gamma^{R}} .
\end{aligned}
$$


Правая часть последнего равенства отличается от $q(u, v)$ на слагаемое

$$
(u, \mathscr{L} v)_{G \backslash G^{R}}+(\mathscr{Q} u, \mathscr{B} v)_{\partial G \backslash \partial G^{R}}-(\mathscr{L} u, v)_{G \backslash G^{R}}-(\mathscr{B} u, \mathscr{Q} v)_{\partial G \backslash \partial G^{R}} .
$$

Ввиду стабилизации коэффициентов оператора $\{\mathscr{L}, \mathscr{B}\}$, в каждом из полуцилиндров $\Pi_{+}^{p}$ коэффициенты оператора $\{\mathscr{L}, \mathscr{B}\}-\left\{L^{p}, B^{p}\right\}$ допускают оценку $O\left(e^{-\gamma|x|}\right)$. Достаточно считать, что $u=U^{p}+w$, где $U^{p}-$ линейная комбинация функций вида $\chi u_{k}$ с носителями в $\Pi^{p}$, а $w \in E$ (см. обозначения перед формулой $(2.5))$. Учитывая, что $u_{k}$ удовлетворяют однородной задаче $(2.4)$, получаем оценку

$$
\{\mathscr{L}-\mu, \mathscr{B}\} u(x)=\left\{L^{p}-\mu, B^{p}\right\} w(x)+\left\{\mathscr{L}-L^{p}, \mathscr{B}-B^{p}\right\} u(x)=O\left(e^{-\gamma R}\right)
$$

при $R \rightarrow+\infty$, равномерную по $x=\left(t^{p}, y^{p}\right) \in \Pi_{+}^{p}$ при $t^{p}>R$. Следовательно, если обе функции $u$ и $v$ имеют вид $U^{p}+w$, то

$$
\begin{gathered}
(u, \mathscr{L} v)_{G \backslash G^{R}}-(\mathscr{L} u, v)_{G \backslash G^{R}}=(u,(\mathscr{L}-\mu) v)_{G \backslash G^{R}}-((\mathscr{L}-\mu) u, v)_{G \backslash G^{R}}=O\left(e^{-\gamma R}\right), \\
(\mathscr{Q} u, \mathscr{B} v)_{\partial G \backslash \partial G^{R}}-(\mathscr{B} u, \mathscr{Q} v)_{\partial G \backslash \partial G^{R}}=O\left(e^{-\gamma R}\right)
\end{gathered}
$$

и величина (4.1) допускает оценку $O\left(e^{-\gamma R}\right)$ при $R \rightarrow+\infty$. Поэтому

$$
\begin{aligned}
& \left(\mathscr{N} u_{j}^{ \pm}, \mathscr{D} u_{k}^{ \pm}\right)_{\Gamma^{R}}-\left(\mathscr{D} u_{j}^{ \pm}, \mathscr{N} u_{k}^{ \pm}\right)_{\Gamma^{R}}=q\left(u_{j}^{ \pm}, u_{k}^{ \pm}\right)+O\left(e^{-\gamma R}\right), \\
& \left(\mathscr{N} u_{j}^{ \pm}, \mathscr{D} u_{k}^{\mp}\right)_{\Gamma^{R}}-\left(\mathscr{D} u_{j}^{ \pm}, \mathscr{N} u_{k}^{\mp}\right)_{\Gamma^{R}}=q\left(u_{j}^{ \pm}, u_{k}^{\mp}\right)+O\left(e^{-\gamma R}\right)
\end{aligned}
$$

и остается лишь учесть (2.6).

Предложение 4.2. Матрица $\mathscr{E}^{R}$ с элементами (2.13) не вырождается ни при каком $R \geqslant R_{0}$, где $R_{0}$ - достаточно большое число.

Доказательство. Предположим, что утверждение неверно. Тогда для любого $R^{0}$ существует такое число $R>R^{0}$, что матрица $\mathscr{E} R$ является вырожденной и функции $\mathscr{U}=\sum_{j} c_{j} u_{j}^{-}$и $\mathscr{V}=\sum_{j} c_{j} v_{j}^{-}$связаны соотношением

$$
\mathscr{D} \mathscr{U}=\mathscr{D} \mathscr{V} \quad \text { на } \Gamma^{R},
$$

где $v_{j}^{-}$- решение задачи $(2.12)$, а $\vec{c}=\left(c_{1}, \ldots, c_{M}\right)$ - некоторый вектор, причем $|\vec{c}|=1$. Согласно уравнению на $\Gamma^{R}$ из $(2.12)$, имеем

$$
\mathscr{N} \mathscr{U}=\mathscr{N} \mathscr{V} \text { на } \Gamma^{R} \text {. }
$$

В формуле Грина (2.9) положим $u=v=\mathscr{V}$. Учтем первые два уравнения (2.12) и формулы $(4.2),(4.3)$ и получим

$$
(\mathscr{N} \mathscr{U}, \mathscr{D} \mathscr{U})_{\Gamma^{R}}-(\mathscr{D} \mathscr{U}, \mathscr{N} \mathscr{U})_{\Gamma^{R}}=0 .
$$

Отсюда и из леммы 4.1 следует, что

$$
0=i \sum_{j}\left|c_{j}\right|^{2}+o(1)=i+o(1) .
$$

Полученное противоречие заканчивает доказательство.

Предложение 4.3. Пусть вектор $a(R)=\left(a_{1}(R), \ldots, a_{M}(R)\right)$ доставляет минимум функиионала $J_{l}^{R}$ из формуль (2.11). Тогда

$$
J_{l}^{R}(a(R))=O\left(e^{-2 \gamma R}\right) \quad \text { npu } R \rightarrow \infty,
$$


где $\gamma$ - число из формулы (2.8). Для всех $R \geqslant R_{0}$ компоненты вектора $a(R)$ равномерно ограничены,

$$
\left|a_{j}(R)\right| \leqslant \text { const }<\infty, \quad j=1, \ldots, M .
$$

Доказательство. Обозначим через $Y_{l}^{R}$ решение задачи $(2.10)$, где в качестве $a_{j}, j=1, \ldots, M$, взяты элементы $S_{l j}$ матрицы рассеяния $S$ задачи $(2.7)$. Поскольку асимптотику (2.8) можно дифференцировать, получаем

$$
\left.(\mathscr{N}+i \zeta \mathscr{D})\left(Y_{l}^{R}-Y_{l}\right)\right|_{\Gamma^{R}}=O\left(e^{-\gamma R}\right) .
$$

Так как разность $Y_{l}^{R}-Y_{l}$ удовлетворяет первым двум уравнениям задачи (3.1) при $f=0$ и $g=0$, то имеет место оценка (3.2) при $u=Y_{l}^{R}-Y_{l}$ :

$$
\left\|\mathscr{D}\left(Y_{l}^{R}-Y_{l}\right) ; L_{2}\left(\Gamma^{R}\right)\right\| \leqslant|\zeta|^{-1}\left\|(\mathscr{N}+i \zeta \mathscr{D})\left(Y_{l}^{R}-Y_{l}\right) ; L_{2}\left(\Gamma^{R}\right)\right\| \leqslant c e^{-\gamma R} .
$$

Вместе с (2.8) это приводит к оценке

$$
J_{l}^{R}\left(S_{l}\right)=\left\|\mathscr{D}\left(Y_{l}^{R}-\left(u_{l}^{+}+\sum_{j=1}^{M} S_{l j} u_{j}^{-}\right)\right) ; L_{2}\left(\Gamma^{R}\right)\right\|^{2} \leqslant c e^{-2 \gamma R}
$$

с постоянной $c$, не зависящей от $R$. Для того чтобы получить (4.5), остается заметить, что $J_{l}^{R}(a(R)) \leqslant J_{l}^{R}\left(S_{l}\right)$.

Перейдем к оценке минимизатора $a(R)$. Обозначим через $Z_{l}^{R}$ решение задачи (2.10), отвечающее вектору $a(R)=\left(a_{1}(R), \ldots, a_{M}(R)\right)$. В формуле Грина $(2.9)$ положим $u=v=Z_{l}^{R}$. Имеем

$$
\left(\mathscr{N} Z_{l}^{R}, \mathscr{D} Z_{l}^{R}\right)_{\Gamma^{R}}-\left(\mathscr{D} Z_{l}^{R}, \mathscr{N} Z_{l}^{R}\right)_{\Gamma^{R}}=0 .
$$

В силу (4.5)

$$
\left\|\mathscr{D}\left(Z_{l}^{R}-\left(u_{l}^{+}+\sum_{j=1}^{M} a_{j}(R) u_{j}^{-}\right)\right) ; L_{2}\left(\Gamma^{R}\right)\right\|=O\left(e^{-\gamma R}\right), \quad R \rightarrow \infty .
$$

Поскольку

$$
\left.(\mathscr{N}+i \zeta \mathscr{D}) Z_{l}^{R}\right|_{\Gamma^{R}}=\left.(\mathscr{N}+i \zeta \mathscr{D})\left(u_{l}^{+}+\sum_{j=1}^{M} a_{j}(R) u_{j}^{-}\right)\right|_{\Gamma^{R}},
$$

из (4.7) получаем

$$
\left\|\mathscr{N}\left(Z_{l}^{R}-\left(u_{l}^{+}+\sum_{j=1}^{M} a_{j}(R) u_{j}^{-}\right)\right) ; L_{2}\left(\Gamma^{R}\right)\right\|=O\left(e^{-\gamma R}\right), \quad R \rightarrow \infty .
$$

Используя (4.7) и (4.8), приведем (4.6) к виду

$$
\left(\mathscr{N} \varphi_{l}, \mathscr{D} \varphi_{l}\right)_{\Gamma^{R}}-\left(\mathscr{D} \varphi_{l}, \mathscr{N} \varphi_{l}\right)_{\Gamma^{R}}=O\left(e^{-\gamma R}\right),
$$

где $\varphi_{l}=u_{l}^{+}+\sum a_{j}(R) u_{j}^{-}$. По лемме 4.1 левая часть равна $-i\left(1-\sum\left|a_{j}(R)\right|^{2}\right)+$ $o(1)$. Итак,

$$
\sum_{j=1}^{M}\left|a_{j}(R)\right|^{2}=1+o(1)
$$


Доказательство теоремы 2.1. Пусть $Y_{l}, Z_{l}^{R}$ и $\left(a_{1}(R), \ldots, a_{M}(R)\right)$ - те же, что в предложении 4.3. Подставим $u=v=U_{l}:=Y_{l}-Z_{l}^{R}$ в формулу Грина (2.9). Поскольку $U_{l}$ удовлетворяет первым двум уравнениям (2.10), имеем

$$
\left(\mathscr{N} U_{l}, \mathscr{D} U_{l}\right)_{\Gamma^{R}}-\left(\mathscr{D} U_{l}, \mathscr{N} U_{l}\right)_{\Gamma^{R}}=0 .
$$

Положим

$$
\varphi_{l}=u_{l}^{+}+\sum_{j=1}^{M} a_{j}(R) u_{j}^{-}, \quad \psi_{l}=u_{l}^{+}+\sum_{j=1}^{M} S_{l j} u_{j}^{-}
$$

и запишем $U_{l}$ в виде

$$
U_{l}=Y_{l}-Z_{l}^{R}=\left(Y_{l}-\psi_{l}\right)+\left(\psi_{l}-\varphi_{l}\right)+\left(\varphi_{l}-Z_{l}^{R}\right) .
$$

Заметим, что $\left.\left(Y_{l}-\psi_{l}\right)\right|_{\Gamma^{R}}=O\left(e^{-\gamma R}\right)$ в силу (2.8). С учетом оценок (4.7), (4.8) и предложения 4.3 это позволяет перейти от (4.9) к соотношению

$$
\left(\mathscr{N}\left(\psi_{l}-\varphi_{l}\right), \mathscr{D}\left(\psi_{l}-\varphi_{l}\right)\right)_{\Gamma^{R}}-\left(\mathscr{D}\left(\psi_{l}-\varphi_{l}\right), \mathscr{N}\left(\psi_{l}-\varphi_{l}\right)\right)_{\Gamma^{R}}=O\left(e^{-\gamma R}\right) .
$$

Здесь левая часть вычисляется непосредственно и равна $i \sum_{j=1}^{M}\left|a_{j}(R)-S_{l j}\right|^{2}+$ $O\left(e^{-\gamma R}\right)$ (достаточно воспользоваться представлениями (4.10) и леммой 4.1). Окончательно получаем

$$
\sum_{j=1}^{M}\left|a_{j}(R)-S_{l j}\right|^{2}=O\left(e^{-\gamma R}\right) .
$$

\section{ЛитеРАТУРА}

[1] С. А. Назаров, Б. А. Пламеневский, Эллиптические задачи в областлх с кусочно гладкой гранищей, Наука, М., 1991.

[2] V. Grikurov, E. Heikkola, P. Neittaanmäki, B. Plamenevskii, On computation of scattering matrices and on surface waves for diffraction gratings, Numer. Math., 94:2 (2003), 269-288.

[3] Б. А. Пламеневский, О. В. Сарафанов, О методе вычисления матрии, рассеяния для волноводов, Алгебра и анализ, 23:1 (2011), 200-231.

[4] М. С. Агранович, М. И. Вишик, Эллиптические задачи с параметром и параболические задачи общего вида, УМН, 19:3 (1964), 53-160.

[5] Ж.-Л. Лионс, Э. Мадженес, Неоднородные граничные задачи и их приложения, М., Мир, 1971.

[6] В. А. Кондратьев, Краевые задачи для эллиптических уравнений в областях с коническими или угловыми точками, Труды ММО, 16 (1967), 219-292.

[7] P. Grisvard, Elliptic Problems in Nonsmooth Domains, Pitman, Boston, 1985.

[8] V. A. Kozlov, V. G. Maz'ya, J. Rossmann, Elliptic Boundary Value Problems in Domains with Point Singularities, Math. Surveys and Monographs, vol. 52, Amer. Math. Soc., Providence, RI, 1997.

[9] Л. Берс, Ф. Джон, М. Шехтер, Уравнения с частными производными, Мир, М., 1966.

Санкт-Петербургский государственный университет e-mail: boris.plamen@gmail.com

Санкт-Петербургский государственный университет

e-mail: saraf@math.nw.ru 